\title{
Clusters of ant colonies and robust criticality in a tropical agroecosystem
}

\author{
John Vandermeer ${ }^{1,2}$, Ivette Perfecto ${ }^{2} \&$ Stacy M. Philpott ${ }^{3}$
}

Although sometimes difficult to measure at large scales, spatial pattern is important in natural biological spaces as a determinant of key ecological properties such as species diversity, stability, resiliency and others ${ }^{1-6}$. Here we demonstrate, at a large spatial scale, that a common species of tropical arboreal ant forms clusters of nests through a combination of local satellite colony formation and density-dependent control by natural enemies, mainly a parasitic fly. Cluster sizes fall off as a power law consistent with a socalled robust critical state $\mathrm{e}^{7}$. This endogenous cluster formation at a critical state is a unique example of an insect population forming a non-random pattern at a large spatial scale. Furthermore, because the species is a keystone of a larger network that contributes to the ecosystem function of pest control, this is an example of how spatial dynamics at a large scale can affect ecosystem service at a local level.

It has been common to assume, sometimes only implicitly, that the patchiness of an ecosystem reflects some underlying habitat factor (for example, marshweed occurs in marshes) even though that factor may not be evident ${ }^{8,9}$. However, it is well known that various intrinsic biological dynamics are capable of producing pattern even in a landscape that is homogeneous for the organism involved ${ }^{10,11}$. This raises the question for any non-random spatial pattern about whether it is caused by factors that are exogenous (broadly, underlying habitat patchiness) or endogenous (broadly, biological aspects of the organism independent of the habitat patchiness ${ }^{12,13}$.

In our study site in southern Mexico the underlying habitat for a species of tropical arboreal ant, Azteca instabilis, is essentially uniform. It is the collection of shade trees planted in a coffee plantation, where the original intention of the farmer was to plant the trees uniformly. The ant species is common in the Mesoamerican tropics, where it is frequently encountered on casual walks in the forest. However, discerning any spatial pattern of its colonies is inevitably obstructed by the heterogeneity of the habitat it normally occupies. However, the species also inhabits shade trees in traditional shaded coffee farms, a uniform habitat both by intention and as measured. Ant nest formation is exclusively in the shade trees; consequently a non-random pattern in the spatial distribution of ant colonies must be a result of endogenous factors, because the underlying habitat is uniform. We find that the colonies indeed are non-random, even though the shade trees themselves are uniform (Fig. 1).

The basic biology of the ant is not unusual. After a queen establishes a colony in a tree, the colony may grow to the point that satellite nests are established in neighbouring trees, presumably one part of the mechanism whereby patchiness is generated. Although the details of satellite formation are not completely known (see Supplementary Information), it is evident from our data that ants within a particular nest establish other nests in nearby trees. Unabated satellite formation would obviously result in a continuous expansion of nests throughout all shade trees in the habitat, which means that some force must limit this expansion. On this farm the ant has a series of natural enemies, any one of which, or any combination thereof, could form the basis for the control that must occur. A parasitoid phorid fly is known to reduce ant foraging activity ${ }^{14}$, and has a
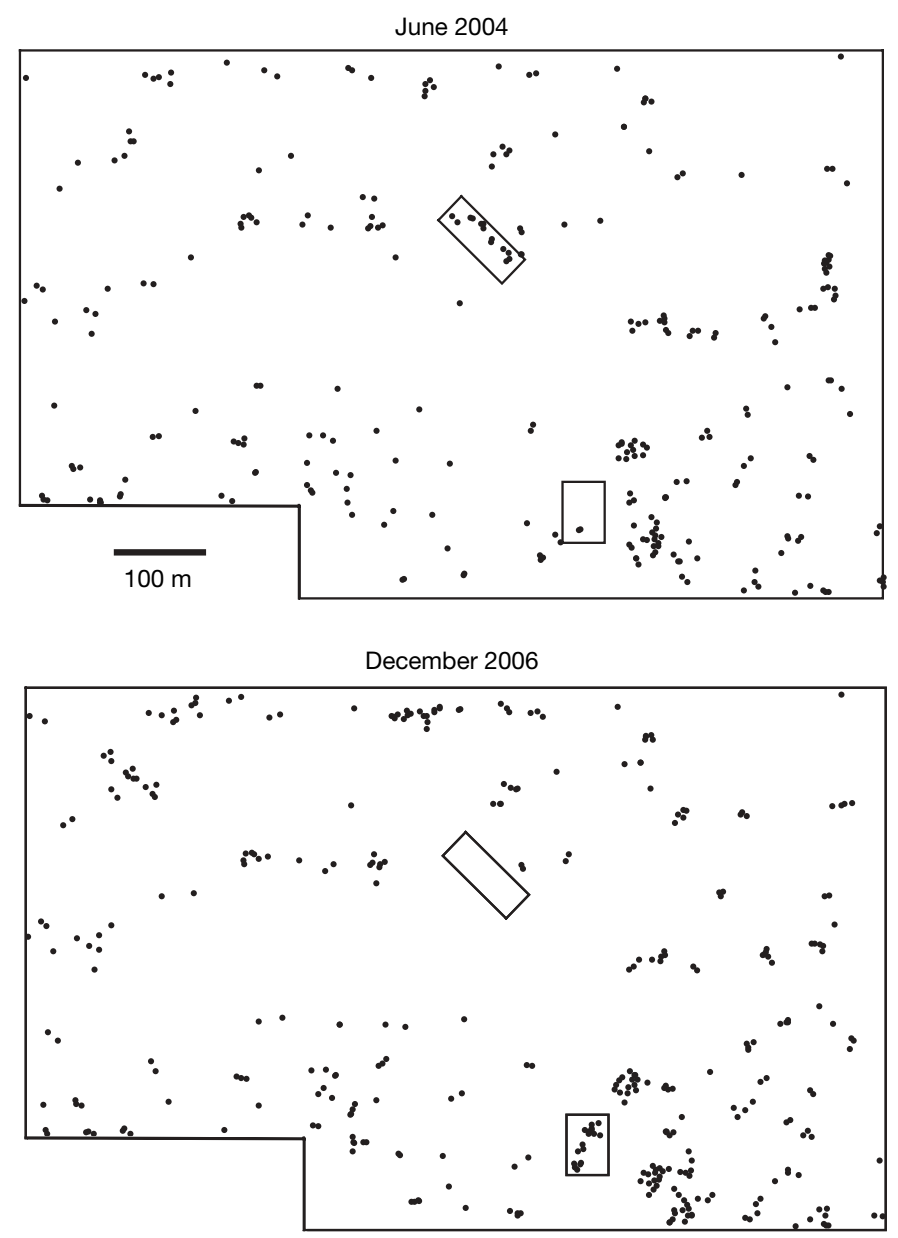

Figure 1 | Distribution of nests of Azteca instabilis over a 45-ha plot (the three missing hectares are on inaccessible terrain). The distributions of colonies found in all censuses are notably clumped (the first and last censuses are shown here) with an average of 328 trees occupied. The nests appear to drift around, as is evident from a comparison of the two panels. Note the dynamic nature of the system over time: the two small rectangles illustrate both the complete disappearance of a cluster and the appearance of another cluster where only a single nest had been before. 
density-dependent response to clusters of ant nests (Fig. 2), in addition to a qualitative behavioural response to the ants (see Supplementary Information).

Based on this natural history, we propose a three part dynamic. First, nuptial flights produce founding queens that disperse as propagule rain over a large area. Second, successful colonies occupy neighbouring trees with satellite colonies. Third, phorid parasitoids concentrate on clusters of ant nests, causing a dramatic behavioural response and possibly direct mortality, thus dramatically reducing ant survivorship in dense clusters of nests. The cellular automata model developed here (see Methods and Supplementary Information) is based on these three features, where a central cell becomes occupied or dies depending on the Moore neighbourhood, $N$, with the probability of satellite expansion being a linear function of $N\left(p_{\mathrm{s}}=s_{0}+s_{1} N\right)$, as is the probability of mortality $\left(p_{\mathrm{m}}=m_{0}+m_{1} N\right)$.

The range of parameter values to instantiate the cellular automata model (see Methods and Supplementary Information) obtained from the field censuses were: for satellite expansion, $s_{0}=0.0-0.8$, $s_{1}=0.0133-0.035$; and for mortality, $m_{0}=0-0.45, m_{1}=0.031-$ 0.097. A systematic search of this range of parameter space produced the following parameters: $s_{0}=0.0035, s_{1}=0.035, m_{0}=0.116$, $m_{1}=0.036$, as those producing the best approximation to both the population densities of nests over time and the cluster size distribution (as measured by the mean/variance ratio). Output from the model and observed data from the field are shown in Fig. 3.

The overall population densities in the simulations are concentrated between 200 and 500, and the mean variance ratios between 0.4 and 0.5 , both close to the range of our observations in nature (represented as horizontal lines in Fig. 3). The model output reflects the erratic nature of cellular automata models, with the same parameters generating a dramatic variability both of population densities and mean variance ratios. However, because the possible range could be from 0 to 10,800 for population density and from 0 to + infinity for mean variance ratios, the ability of the model, with parameter values within our empirical envelope, to generate population densities and patterns so close to those we observed in the field suggests that the basic interpretation of the spatial dynamics is probably correct.

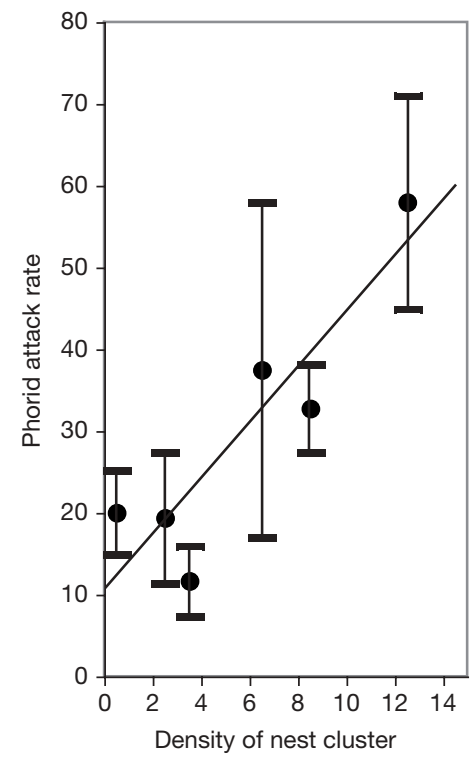

Figure 2 | Attack rates of phorid fly parasites as a function of nest-cluster density. Density of nests based on a $20 \mathrm{~m}$ circle surrounding the point at which phorid attack trials were done. Error bars, s.e.m. The attacks were highly variable, but the relation with the density of the local clusters of ant nests is statistically significant $(P=0.042$; see also Supplementary Information).
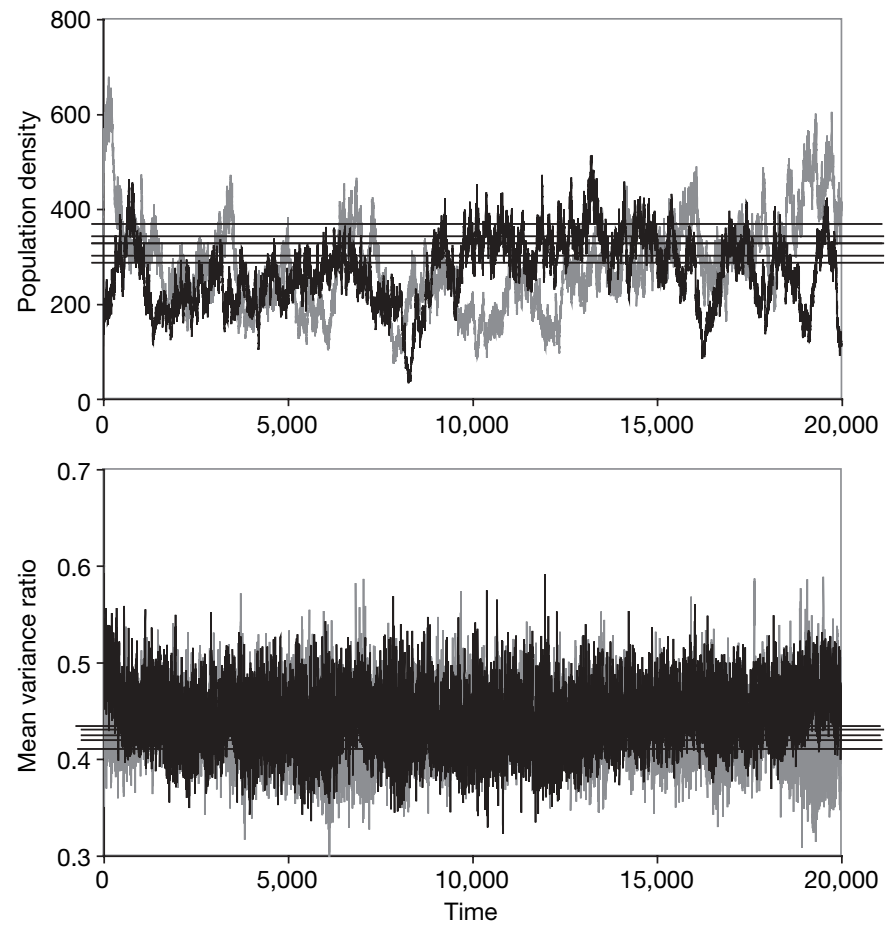

Figure 3 | Time (in six month intervals) series for population density (top) and mean variance ratios (bottom) for the parameters $s_{0}=0.0035$, $s_{1}=0.035, m_{0}=0.116$ and $m_{1}=0.036$. In both cases two separate runs are pictured: one in black, the other in grey (hardly noticeable in the bottom panel because the two runs are so similar). Horizontal lines are the values of the six field samples (two samples are so close as to appear the same).

Although our modelling approach is distinct, the underlying biological interactions are similar to those studied by Pascual and colleagues, suggesting that we should expect a power law relation between cluster size and frequency. Indeed, as expected, the distribution of cluster sizes in our plot does follow a power function (Fig. 4a). Furthermore, calculating the frequency of cluster sizes, as generated by the model, produces a similar power relation (Fig. 4b) as would be suggested if the system is near criticality ${ }^{15}$. Further studies of the model show that there is a broad region of parameter space in which the power law holds, suggesting that this may be a case of robust criticality ${ }^{16}$.
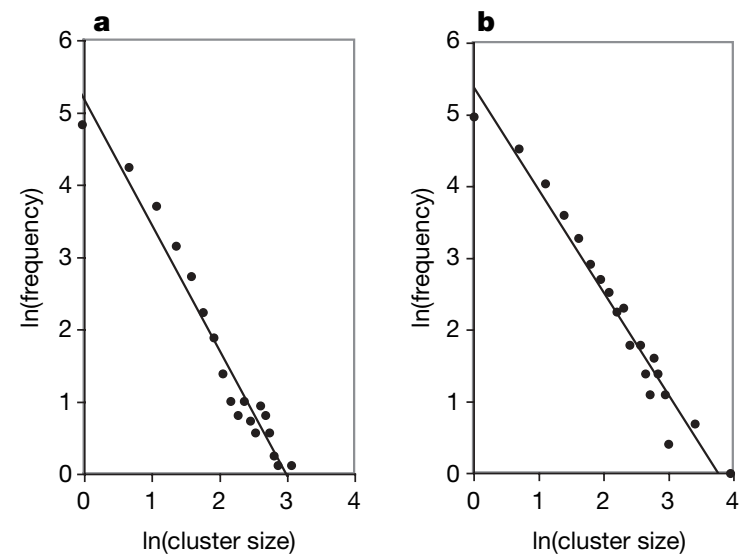

Figure 4 | Log of cumulative frequency of log cluster sizes. a, From field samples, based on a minimum distance of $20 \mathrm{~m}$ between nests that are judged to be in the same cluster. $\mathbf{b}$, From field-parameterized cellular automata stochastic model, based on a $90 \times 120$ lattice, where each lattice point is intended to model a single shade tree. Clusters are defined based on individual lattice points in contact with any other lattice point in the Moore neighbourhood. 
The importance of these results lies in the fact that strong spatial pattern is formed in the face of habitat homogeneity, connecting with the well-known consequences of spatial pattern on topics such as species diversity, ecosystem stability or resiliency and others. Furthermore, it is likely that this pattern formation also is related to biological control of several important coffee pests such as the coffee berry borer (Hypthenemus hampei) ${ }^{17}$, the green coffee scale (Coccus viridis) $)^{18}$ and coffee rust (Hemileia vastatrix) ${ }^{19}$. All of these controls are effected through the spatial patterning of this system, as discussed in detail elsewhere ${ }^{20}$.

\section{METHODS SUMMARY}

All trees in a 45-ha plot in a shaded coffee plantation were located and the presence of ant colonies therein noted, a process repeated at 6-month intervals for 2 years. Non-randomness of nests was established through quadrat-based computation of mean and variance of number of nests per quadrat. Attack rates of phorid flies were determined in locations that varied in the local number of ant nests, by placing a small amount of ant-nest carton with a few ants in a container and counting the number of phorid attacks over a $20 \mathrm{~min}$ period. The stochastic cellular automata was constructed based on the natural history observations of the ants forming satellite nests on nearby trees plus the phorid flies encountering nests in proportion to their local density. The model was parameterized with field data and compared with actual distributions in the field. Both the distribution of nests in the field and the distribution generated by the cellular automata were examined for the distribution of cluster sizes.

Full Methods and any associated references are available in the online version of the paper at www.nature.com/nature.

\section{Received 21 August; accepted 13 November 2007.}

1. Kefi, S. et al. Spatial vegetation patterns and imminent desertification in Mediterranean arid ecosystems. Nature 449, 213-217 (2007).

2. Ball, P. The Self-made Tapestry: Pattern Formation in Nature. (Oxford Univ. Press, Oxford, 1999).

3. Durrett, R. \& Levin, S. Spatial aspects of interspecific competition. Theor. Pop. Biol. 53, 30-43 (1998)

4. Czárán, T. \& Bartha, S. Spatiotemporal dynamic models of plant populations and communities. Trends Ecol. Evol. 7, 38-42 (1992).

5. Gurney, W. S. C. \& Veitch, A. R. Self-organization, scale and stability in a spatial predator-prey interaction. Bull. Math. Biol. 62, 61-86 (2000).

6. Levin, S. A. The problem of pattern and scale in ecology: the Robert H. MacArthur Award Lecture. Ecology 73, 1943-1967 (1992)
7. Pascual, M., Manojit, R., Guichard, F. \& Flierl, G. Cluster size distributions: signatures of self-organization in spatial ecologies. Phil. Trans. R. Soc. Lond. B 357, 657-666 (2002).

8. Turner, S. J. O’Neill, R. V., Conley, W., Conley, M. R. \& Humphries, H. C. in Quantitative Methods in Landscape Ecology (eds Turner, M. G. \& Gardner, R. H.) (Ecological Studies, Vol. 82) 17-49 (Springer, New York, 1990).

9. Forman, R. T. T. \& Gordon, M. Landscape Ecology (Wiley, New York, 1987).

10. Hassell, M. P., Comins, H. N. \& May, R. M. Species coexistence and self-organizing spatial dynamics. Nature 370, 290-292 (1994)

11. Reitkerk, M., Dekker, S. C., de Ruiter, P. C. \& van de Koppel, J. Self-organized patchiness and catastrophic shifts in ecosystems. Science 305, 1926-1929 (2004).

12. Wooton, J. T. Local interactions predict large-scale pattern in empirically derived cellular automata. Nature 413, 841-844 (2001).

13. Maron, J. L. \& Harrison, S. Spatial pattern formation in an insect host-parasitoid system. Science 278, 1619-1621 (1997).

14. Philpott, S. M., Maldonado, J., Vandermeer, J. \& Perfecto, I. Taking trophic cascades up a level: behaviorally-modified effects of phorid flies on ants and ant prey in coffee agroecosystems. Oikos 105, 141-147 (2004).

15. Pascual, M. \& Guichard, F. Criticality and disturbance in spatial ecological systems. Trends Ecol. Evol. 20, 23-27 (2005).

16. Roy, M., Pascual, M. \& Franc, A. Broad scaling region in a spatial ecological system. Complexity 8, 19-27 (2003).

17. Perfecto, I. \& Vandermeer, J. The effect of an ant/scale mutualism on the management of the coffee berry borer (Hypothenemus hampei) in southern Mexico. Agric. Ecosyst. Environ. 117, 218-221 (2006).

18. Vandermeer, J. \& Perfecto, I. A keystone mutualism drives pattern in a power function. Science 311, 1000-1002 (2006).

19. Vandermeer, J., Perfecto, I. \& Liere, H. Evidence for effective hyperparasitism on the coffee rust, Hemileia vastatrix, by the insect pathogen, Lecanicillium (Verticillium) lecanii through a complex ecological web. J. Agric. Sci. (submitted).

20. Perfecto, I. \& Vandermeer, J. H. Spatial pattern and ecological process in the coffee agroecosystem. Ecology (in the press).

Supplementary Information is linked to the online version of the paper at www.nature.com/nature.

Acknowledgements We thank S. Levin and M. Reitkerk for reading an earlier version of the manuscript, and M. Pascual for advice. We also thank J. Maldonado, B. Estaban Chilel and G. López-Bautista, who performed the bulk of the field censuses, and the Peter's Foundation for permission to establish the plot on Finca Irlanda, and for logistic support. El Colegio de la Frontera Sur (ECOSUR), especially G. Ibarra Núñez and A. Garcia-Ballinas, also provided logistical support. This work was supported by a National Science Foundation grant to I.P. and J.V.

Author Information Reprints and permissions information is available at www.nature.com/reprints. Correspondence and requests for materials should be addressed to J.V. (jvander@umich.edu). 


\section{METHODS}

The system. The site is located at Finca Irlanda, a 300-ha organic coffee farm in the Soconusco region of Chiapas, Mexico $\left(15^{\circ} 11^{\prime} \mathrm{N}, 92^{\circ} 20^{\prime} \mathrm{W}\right)$, established over 100 years ago (see Supplementary Information for a more detailed site description).

Field surveys of ant nests. A $600 \mathrm{~m} \times 800 \mathrm{~m}$ plot ( $45 \mathrm{ha}$ ) was established within which all shade trees were located and mapped to the nearest $2 \mathrm{~m}$ (usually to the nearest $1 \mathrm{~m}$, but at times the terrain became too difficult to manoeuvre so we relaxed the precision in those areas). The first census was during the summer (May-September) of 2004; there were five subsequent censuses in May and December 2004, 2005, and December 2006. Only 45 of the 48 ha (from a $600 \mathrm{~m} \times 800 \mathrm{~m}$ plot) were surveyed because three of the hectares in one corner were located next to a cliff that was inaccessible. Note that our definition of a 'colony' or 'nest' in a tree is based on the behaviour of the ant. Upon disturbance, many workers swarm out of the tree in an obvious collective defence, and it is never questionable whether there is a nest. The second to sixth censuses used the coordinates from the first census to locate every tree in the 45-ha plot and, rapping the tree sharply with a stick, evidence of disturbed workers of $A$. instabilis was recorded.

Field surveys of phorid attack rates. Artificial ant nests, consisting of small pieces of carton from a natural nest plus about 50 individual ants, were placed in small plastic containers with fluon painted on the upper margins and taken into the field with a tight-fitting cover in place to avoid odour release on the trip to the field. Phorid density was assessed by opening the top of the container and recording each parasitoid attack on a worker ant, during a period of 5 minutes. Because other species of phorids and small flies are common in the area, only an actual attack was registered if an individual of Pseudacteon sp. attacked A. instabilis. Eighteen sites were selected from the 45-ha plot, covering a range of local cluster sizes (see Supplementary Information), and phorid attacks were recorded at four locations within the general area of the cluster of ant nests. This sampling was done in August 2006.
The model. A $120 \mathrm{~m} \times 90 \mathrm{~m}$ grid was established on a torus. Each point in the grid was meant to be a single shade tree and was either occupied (1) or not (0) by a colony. A cellular automata approach was used with the Moore neighbourhood (eight surrounding cells) acting as the 'clump size'. This established the satellite colonies. The probability of extinction was tied to the expected likelihood that phorid flies would attack, which, in turn, is taken to be linearly related to the local population density of colonies (as supported by our phorid census). Finally, because the field surveys revealed new occupancies always associated with previously occupied nests, we assumed that the probability of a new nest from a nuptial queen was close to zero, and thus was ignored in the model.

Formally, let $x(i, j)$ be a binary variable located at the grid point $\{i, j\}$. We define the local population density at that grid point as $N(i, j)$, the sum of the binary variables in the Moore neighbourhood of that grid point. The overall population density is $N_{\mathrm{T}}=\sum_{i} \sum_{j} x(i, j)$. At each iteration of the model, if $x(i, j)=0$ and $N(i, j)>0$, a random number, $R_{1}$, is drawn between 0 and 1 ; and if $R_{1}<s_{0}+s_{1} N(i, j)$, then, $x(i, j)=1$, otherwise $x(i, j)=0$. Note that because $R_{1}$ is assessed only if $x(i, j)=0$ and $N(i, j)>0$, the possibility of an isolated colonization is excluded. Thus $s_{0}$ and $s_{1}$ are the parameters of 'clump expansion through the creation of a satellite nest' referred to henceforth as simply 'satellite expansion'. Also at each iteration, to calculate the probability of phorid attack, a random number, $R_{2}$, is drawn between 0 and 1 , and if, for all $x(i, j)=1$, $R_{2}<m_{0}+m_{1} N(i, j)$, then $x(i, j)=0$, otherwise $x(i, j)=1$. Thus, $m_{0}$ and $m_{1}$ are the parameters of phorid attack.

Updating of $x$ is cell by cell, and final population densities were calculated at the end of the iteration. In all simulations the system was evidently in a relatively stable state, in terms of total population density and qualitative pattern of nests on the grid, after about 400 iterations. Thus all simulations reported are of the system after discarding the first 450 iterations. Further analytical details of this model are currently under study and will be reported elsewhere. Here we only seek to demonstrate its consistency with field data.

Parameters for the model were estimated from the field data, with a process explained in detail in the Supplementary Information. 